\title{
1 Mitonuclear interactions affect locomotor activity and sleep in
}

\section{Drosophila melanogaster}

3

4 Lucy Anderson ${ }^{1}$, M. Florencia Camus ${ }^{2}$, Katy M. Monteith ${ }^{1}$, Tiina S. Salminen ${ }^{1,3}$, Pedro F. Vale ${ }^{1}$

5

$6{ }^{1}$ Institute of Evolutionary Biology, School of Biological Sciences, University of Edinburgh, UK.

$7 \quad{ }^{2}$ Research Department of Genetics, Evolution and Environment, University College

8 London, Gower Street, London, WC1E 6BT, United Kingdom

9

$10 \quad{ }^{3}$ Faculty of Medicine and Health Technology, Tampere University, Finland

11 


\section{Abstract}

13

14 Mitochondria are organelles that produce cellular energy in the form of ATP through oxidative

15 phosphorylation, and this primary function is conserved between many taxa. Locomotion is a

16 trait that is highly reliant on metabolic function and expected to be greatly affected by

17 disruptions to mitochondrial performance. To this end, we aimed to examine how activity and

18 sleep vary between Drosophila melanogaster strains with different geographic origins, how these

19 patterns are affected by mitochondrial DNA (mtDNA) variation, and how breaking up co-

20 evolved mito-nuclear gene combinations affect the studied activity traits. The results

21 demonstrate that Drosophila strains from different locations differ in sleep and activity, and the

22 extent of variation differs between sexes, females in general being more active. By comparing

23 activity and sleep of $\mathrm{mtDNA}$ variants introgressed onto a common nuclear background in

24 cytoplasmic hybrid (cybrid) strains, we establish that mtDNA variation affects both traits, sex

25 specifically. Furthermore, by using previously published mtDNA copy number data, we detected

26 a positive correlation between mtDNA copy number and the activity levels of the cybrid flies.

27 Altogether, our study shows that both mtDNA variation and mitonuclear interactions affect

28 activity and sleep patterns, highlighting the important role that both genomes play on life-history

29 trait evolution.

31 Key-words: co-evolution; cybrid; fruit fly; mitochondria; mtDNA; mtDNA copy number;

32 OXPHOS; variation 


\section{Introduction}

35 Mitochondria are key organelles in a range of critical metabolic processes and are the primary

36 energy producers for the eukaryotic cell. In addition to this primary role, they are also involved

37 in a range of other vital processes that control key aspects of cellular growth and regulation such

38 as signalling (1), cellular differentiation (2), cell death (3) and immunity (4). The mitochondrial

39 machinery responsible for ATP production via oxidative phosphorylation (OXPHOS) is jointly

40 encoded by the mitochondrial (mtDNA) and nuclear genomes. All the thirteen protein coding

41 genes of the mtDNA genome are involved with OXPHOS, as majority of the OXPHOS

42 subunits as well as over 1200 other genes required for mitochondrial functions are encoded by

43 nuclear DNA (5-8). In addition to vast differences in genome size, there are large differences in

44 genome copies, with hundreds of mtDNA copies inhabiting each diploid cell (9). Consequently,

45 precise and synchronised coordination between the two genomes is required for proper assembly

46 and function of the components of the electron transport chain and mitochondrial functions.

47 Disruptions to this system - by mutations in the mitochondrial or nuclear counterparts - can

48 have consequences to a wide-range of life-history phenotypes (10), and in severe cases leads to

49 mitochondrial disease (11-15).

51 A consequence of this tight intergenomic partnership is that any trait with heavy metabolic

52 underpinnings is reliant on the compatibility between mitochondrial and nuclear genomes. Two

53 such traits which are vital for everyday function are locomotor activity and sleep. Sleep is

54 integral to regular brain function, influencing processes such as learning and memory (16), and

55 plays a role in cellular processes such as metabolic recovery and oxidative stress (17). Continued

56 sleep deprivation results in fatality for both invertebrate and vertebrate species (18-20). In

57 humans, sleep deprivation is associated with an increased risk of metabolic and cognitive

58 disorders (21). Locomotion is also heavily reliant on metabolic function, with studies finding a

59 strong positive correlation between activity and resting metabolic rate (22). Moreover, deviations 
60 of optimal organismal activity as well as exercise intolerance have been linked to several

61 metabolic disorders originating from mitochondrial dysfunction (23). For instance, Drosophila

62 models of neuromuscular degeneration have shown a progressive decrease in fly activity with

63 time (24). Additionally, nutritional metabolic interventions in the form of dietary restriction have

64 shown to change activity patterns (25).

65

66 Given their tight link with metabolism, mutations affecting mitochondrial function are predicted

67 to affect both sleep and locomotor activity. A previous review of primary mitochondrial diseases

68 described sleep disorders in humans are associated with mutations in mitochondrial DNA (26).

69 However, there are currently few clear causal links between specific mitochondrial mutations and

70 disrupted activity and sleep patterns $(26,27)$. The fruit fly, Drosophila melanogaster, offers a

71 powerful system to address the link between mitochondrial variation and activity and sleep

72 disruption. Drosophila is an established genetic model system, including the study of mito-nuclear

73 effects on various phenotypic traits (28-31). As mtDNA is maternally inherited, introgression

74 enables the generation of flies with specific combinations of nDNA and mtDNA. Generation of

75 cytoplasmic hybrid (cybrid) lines, in which flies contain a mtDNA variant on a controlled nuclear

76 background, allows the effects of mtDNA mutations disentangled from nuclear genome

77 variation (32). A large body of work using Drosophila cybrid lines has established that several

78 measures of life-history phenotypes are modulated by changes of the mitochondrial genome,

79 including aging (33-35), fitness $(31,36,37)$, and metabolic rate (38).

81 Drosophila is also an established model for the study of sleep and circadian rhythms and display a

82 state of quiescence which shares critical features of mammalian sleep (39). These similarities

83 include an elevated arousal threshold (39), altered brain electrical activity (40) and a decrease in

84 amount of sleep as flies age (41). Furthermore, gene expression associated with 'waking' in fruit

85 flies has been shown to correlate with 'waking' genes in mammals (39). A relevant example is 
86 the mtDNA-encoded Cytochrome oxidase C, subunit I, which has been demonstrated to have

87 elevated expression during the initial hours following sleep in both Drosophila and rats (39). This

88 homology between Drosophila and mammalian sleep, combined with the knowledge that many of

89 the genetic and molecular regulators of sleep are conserved between flies and humans (42), has

90 prompted extensive use of the fruit fly as a genetically tractable model organism in the study of

91 sleep.

92

93 To specifically address the role of variation in the mitochondrial DNA on activity and sleep, we

94 examined the sleep-wake cycles and activity profiles of a worldwide collection of eight $D$.

95 melanogaster lines, in addition to a set of derived cybrid lines which contained each of the eight

96 mtDNA variants introgressed onto a single common nuclear background. This experimental

97 setup allowed us to investigate the baseline activity and sleep profiles of each line and to assess

98 the contribution of mtDNA to these phenotypes. Further, because the mitochondrial genome of

99 each line has been previously characterized (37) and presents unique mtDNA variation at the

100 haplotype level as well as common variants at the haplogroup level, we were able to associate this

101 variation on sleep and activity patterns, sex specifically.

102

103 


\section{Materials and methods}

\section{Fly strains, backcrossing and rearing conditions}

106 We sourced eight wild-type D. melanogaster strains, with distinct geographic origins (Table 1),

107 originally obtained from the Drosophila Stock Center (Bloomington, IN). Cybrid lines were

108 created earlier by backcrossing females from each strain (carrying unique mtDNA variant) to

109 males from the nuclear-donor strain of the Oregon RT strain (Oregon R strain maintained long-

110 term in Tampere, Finland; ORT) for at least 12 generations (37). This resulted in a total of 16

111 strains; 8 of which were the Bloomington derived strains representing coevolved mito-nuclear

112 combinations and 8 were nORT mtDNAx cybrids. All lines were cultured on standard Lewis

113 medium (43), supplemented with yeast, under 12: 12 light: dark cycles at $25^{\circ} \mathrm{C}$ and $60 \%$

114 humidity. Flies were propagated by placing 2-4-day old females and males on food vials for 3-4

115 days, with adults being discarded and egg clutched kept. This rearing regime maintained egg

116 densities low enough to prevent larval overcrowding.

117

\begin{tabular}{|c|c|c|c|c|c|}
\hline Strain & $\begin{array}{l}\text { mtDNA } \\
\text { haplogrou } \\
\mathrm{p}\end{array}$ & $\begin{array}{l}\text { GenBank } \\
\text { accession } \\
\text { number }\end{array}$ & Geographic origin & Latitude & $\begin{array}{l}\text { Amino acid replacement } \\
\text { (OXPHOS complex, } \\
\text { gene) }\end{array}$ \\
\hline ORT & $\mathrm{I}$ & KY559383 & - & - & - \\
\hline KSA2 & $\mathrm{I}$ & KY559384 & $\begin{array}{c}\text { Kariba Dam, } \\
\text { Zimbabwe }\end{array}$ & $17^{\circ} \mathrm{S}$ & $\begin{array}{c}\mathrm{D}_{21} \mathrm{~N} \text { (III, CYTB) } \\
\mathrm{A}_{75} \mathrm{~T} \text { (IV, COIII) }\end{array}$ \\
\hline BOG1 & $\mathrm{I}$ & KY559386 & Bogota, Colombia & $4^{\circ} \mathrm{N}$ & $\mathrm{A}_{356} \mathrm{~T}(\mathrm{I}, \mathrm{ND} 5)$ \\
\hline $\begin{array}{l}\text { WT5 } \\
\text { A }\end{array}$ & $\mathrm{I}$ & KY559387 & $\begin{array}{c}\text { Red Top Mountain, } \\
\text { GA, USA }\end{array}$ & $34^{\circ} \mathrm{N}$ & - \\
\hline M2 & $\mathrm{I}$ & KY559388 & Australia & $25^{\circ} \mathrm{S}$ & $\begin{array}{l}\mathrm{M}_{280} \mathrm{~V}(\mathrm{I}, N D 2) \\
\mathrm{V}_{161} \mathrm{~L}(\mathrm{I}, N D 4) \\
\mathrm{A}_{106} \mathrm{~T}(\mathrm{IV}, C O I) \\
\mathrm{M}_{185} \mathrm{I}(\mathrm{V}, A T P 6)\end{array}$ \\
\hline PYR2 & II & KY559389 & Pyrenees, Spain & $43^{\circ} \mathrm{N}$ & $\begin{array}{c}\mathrm{N}_{114} \mathrm{D}(\mathrm{I}, N D \sigma) \\
\mathrm{V}_{264} \mathrm{M}\left(\mathrm{III}, C Y^{T} B\right)\end{array}$ \\
\hline LS & II & KY559390 & Lausanne, Switzerland & $46^{\circ} \mathrm{N}$ & $\begin{array}{c}\mathrm{S}_{59} \mathrm{~F}(\mathrm{I}, \text { ND5) } \\
\mathrm{D}_{13} \mathrm{~N}(\mathrm{IV}, \text { COIII })\end{array}$ \\
\hline BS1 & II & KY559391 & Barcelona, Spain & $41^{\circ} \mathrm{N}$ & $\mathrm{G}_{58} \mathrm{~S}(\mathrm{IV}, \mathrm{COII})$ \\
\hline
\end{tabular}

118 Table 1. Drosophila melanogaster strains used in the study. Strains were obtained from

119 Bloomington stock centre and originally collected from different continents. GenBank accession numbers refer to the

$120 m t D N A$ coding region sequences. Haplotype-specific amino acid replacements have been added here based on

121 Salminen et al. 2017. 


\section{Experimental design}

123 Tubes for the Drosophila Activity Monitor were prepared prior to the experiments. A solution

124 of 16\% sucrose: $2 \%$ agar was prepared in distilled water and autoclaved for sterilisation and

125 stored at room temperature before re-melting for use. Each clean class tube (Trikinetics,

126 Waltham, MA) contained approximately $1 \mathrm{~cm}$ sucrose-agar medium and a rubber cap in one end

127 of the tube and after the anesthetised fly had been inserted into the tube it was closed with a

128 rubber cap containing a small hole for ventilation (44).

130 Flies from each coevolved and cybrid strain were collected upon eclosion and kept in food vials for

1312 days to make sure flies entering the experiment were mated. Flies were then anesthetised with

$132 \mathrm{CO}_{2}$, sorted by sex and transferred into an activity tube using a fine paintbrush. Fifteen

133 individuals per sex and strain were placed in the tubes and randomly positioned in the activity

134 monitors. Each monitor had a null or blank control in which a recorded position contained an

135 empty tube or no tube, respectively. Monitoring of fly activity and sleep lasted for three adjacent

136 days. The assay was split across five experimental blocks, to allow the inclusion of a subset of

137 replicates for each line in all blocks. Each tube was bisected by an infrared beam and locomotor

138 activity movement was recorded whenever a fly broke the beam. The number of activity counts

139 (beam breaks) per minute was stored. In order to quantify sleep from the activity data generated

140 by the DAM, sleep was defined as a 5 minute time-bin with no registered activity (45). To

141 determine whether individuals/flies are more active simply because they sleep less, we calculated

142 the proportion of time each replicate spends sleeping, as well as the mean activity count whilst

143 awake. The experiments were run under 12: 12 light: dark cycles at $25^{\circ} \mathrm{C}$ at constant temperature

144 and humidity. 


\section{Statistical analysis}

147 Total activity count, mean awake activity and proportion of time sleeping were calculated for

148 each individual fly (see also (46)). Any fly that died during the experiment was excluded from

149 analysis. We analyzed the co-evolved and cybrid lines separately, using otherwise identical

150 statistical models. Total activity count and mean awake activity were analysed using linear

151 mixed effects models (LME). Models fitted 'line', 'sex' and their interaction as categorical

152 fixed effects. Variation in proportion of time asleep was analysed using generalized linear

153 effects models (GLME) assuming binomial distributed error, which also fitted 'line', 'sex' and

154 their interaction as categorical fixed effects. All models included the random effect of

155 'replicate' nested within 'block' to account for variation between individuals among different

156 blocks. We also investigated if breaking up co-evolved mito-nuclear gene complexes affected

157 each of these behavioral outputs. This analysis therefore included the co-evolved and cybrid

158 fly lines to analyse the effect of mtDNA variant on its original (coevolved) or new (cybrid)

159 nuclear background. Similar model structure was used as described above for each response

160 variable, where each model fitted 'type' (coevolved or cybrid), 'sex' and their interactions as

161 categorical fixed effects, and 'line' as a random effect nested within type.

163 mtDNA copy number data of three-day old females and males, measured from the whole adult

164 extracts by Salminen et al. (2017) was used to test the correlation between copy number and

165 activity and sleep patterns. mtDNA copy number was originally measured with qPCR by

166 measuring the ratio between nuclear and mitochondrial target genes (37). Nonparametric

167 Spearman's rank correlation was calculated based on the average mtDNA copy number values

168 and the averages of total activity levels, activity when awake and the proportion flies spent

169 sleeping. Spearman's rank correlation between mtDNA copy number and the studied activity

170 traits were measured in each sex for either the co-evolved or the cybrid lines. $\mathrm{R}$ version 1.1.4

171 (47) was used for analysis and plots, using packages ggplot2 (48), dplyr (49), lme4 (50), car (51) 
bioRxiv preprint doi: https://doi.org/10.1101/2021.10.30.464953; this version posted November 1, 2021. The copyright holder for this preprint

(which was not certified by peer review) is the author/funder, who has granted bioRxiv a license to display the preprint in perpetuity. It is made available under aCC-BY 4.0 International license.

172 and plotrix (52). All datasets and full $\mathrm{R}$ code for all analyses can be found at

173 https://doi.org/10.5281/zenodo.5573904.

174

175 


\section{Results}

\section{Co-evolved fly lines show sex-specific natural variation in sleep and activity patterns}

178 We first evaluated the sleep and activity patterns of genetically and geographically diverse fly

179 lines carrying coevolved mito-nuclear combinations (Table 1). Activity profiles showed that all

180 lines were crepuscular, exhibiting a peak of activity at the onset of the dark period (Fig S1). In

181 the majority of strains, females were significantly more active than males, although the extent of

182 this difference was influenced by genotype and in lines BS1 and WT5A, males were more active

183 than females (Table 2 'Line x Sex' effect; Figure 1A). Overall, males spent a higher proportion of

184 time asleep compared to females, and the extent of this difference varied between genetic

185 backgrounds (Table 2 'Line x Sex' effect; Figure 1B-C). Notably, while males slept for a greater

186 proportion of the day, they were significantly more active while awake compared to females, and

187 again the extent of this variation differed between lines (Table 2 'Line x Sex' effect; Figures 1B-

188 C). This suggests that although females exhibit higher total activity, this is due to females

189 spending more time awake (sleeping less) rather than having higher levels of activity when

190 awake.

\section{Mitochondrial genome affects activity and sleep patterns of the cybrid lines}

193 By introgressing the eight mtDNA variants onto a common nuclear background (ORT), we were

194 able to evaluate how much of the variance in locomotor activity was affected by the mtDNA and

195 newly created mito-nuclear combinations. In general, comparing total activity counts showed

196 that as observed with co-evolved lines, females of each cybrid line were significantly more active

197 than cybrid males, and the extent of this variation was mtDNA specific (Table 2 'Line x Sex'

198 effect; Figure 2A). Males were found to sleep for a significantly larger proportion of time,

199 although unlike the co-evolved lines, the female cybrids were generally more active when awake

200 when compared to males (Figure 2B-C). Both of these differences were also mediated by

201 mtDNA variation (Table 2 'Line $x$ Sex' effect; Figure 2B-C). Therefore, when the effects of 
202 individual mitochondrial genomes are isolated on a common nuclear background females are

203 both more active while awake and also spend less time sleeping than males.
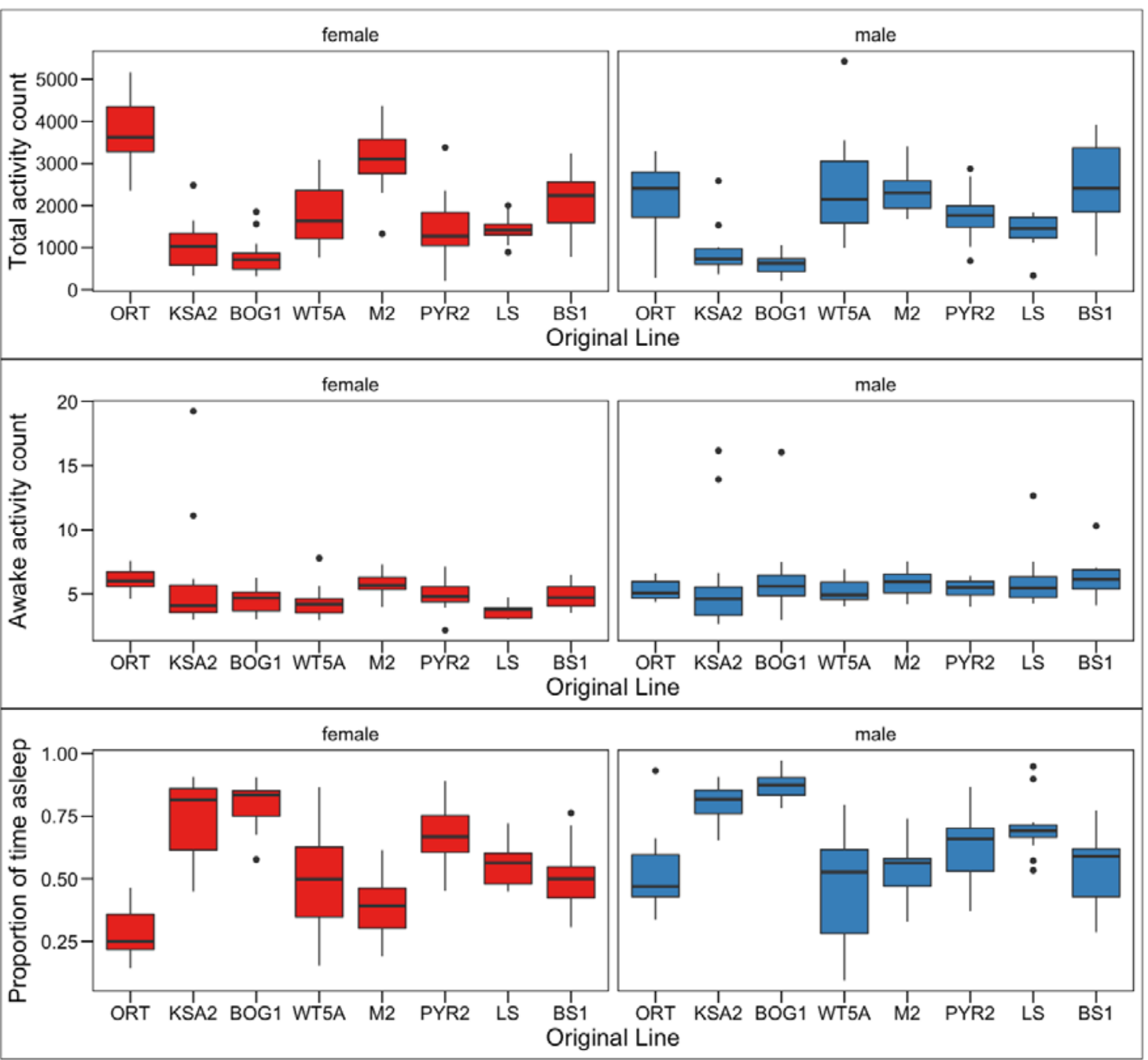

205 Figure 1. Locomotor activity and sleep in females (red) and males (blue) of the coevolved fly

206 lines. A) total number of activity events recorded over three days. B) the total number of activity

207 events recorded when the flies were awake. C) The proportion of time that flies were

208 determined to be asleep, defined as 5 min of inactivity, See Fig S1 for individual actograms and

209 Table 1 for details of each line. See Table 2 for outputs of statistical models. 

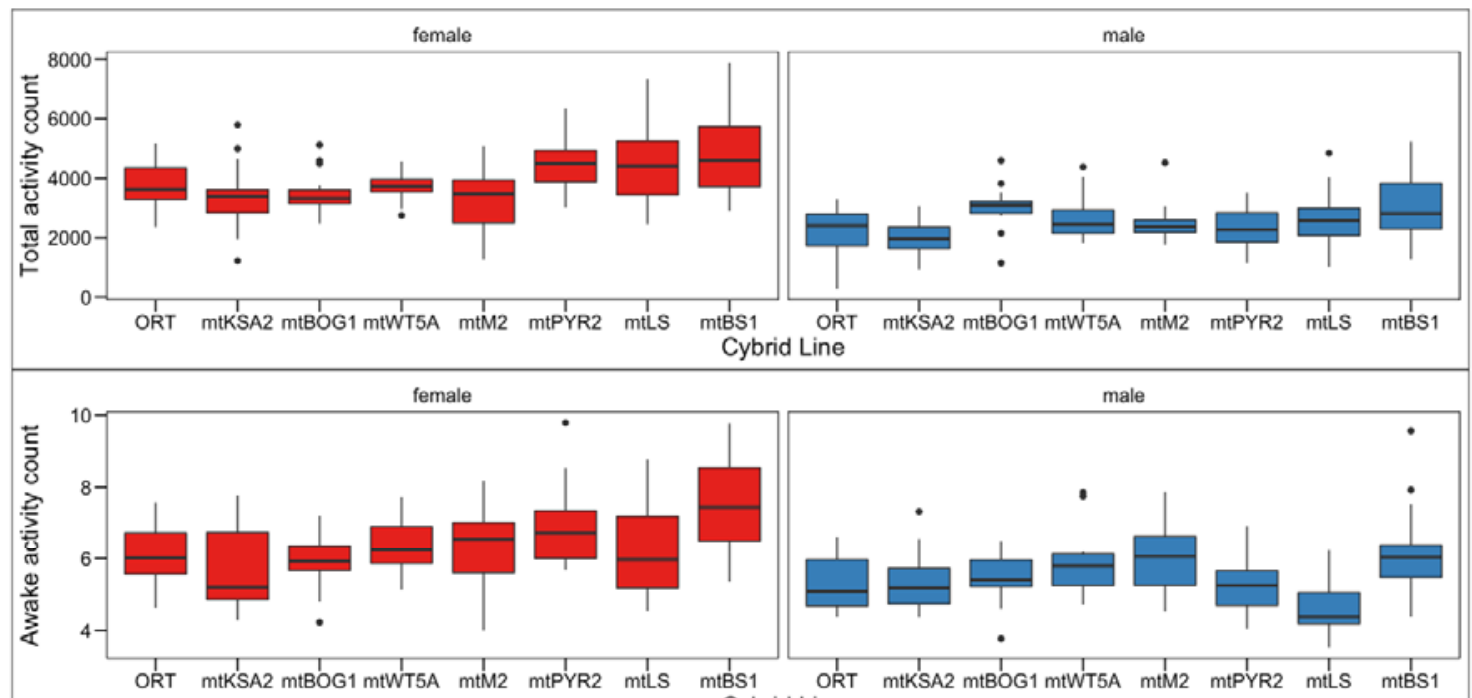

ORT mtKSA2 mtBOG1 mtWT5A mtM2 mtPYR2 mtLs mtBS1 Cybrid Line

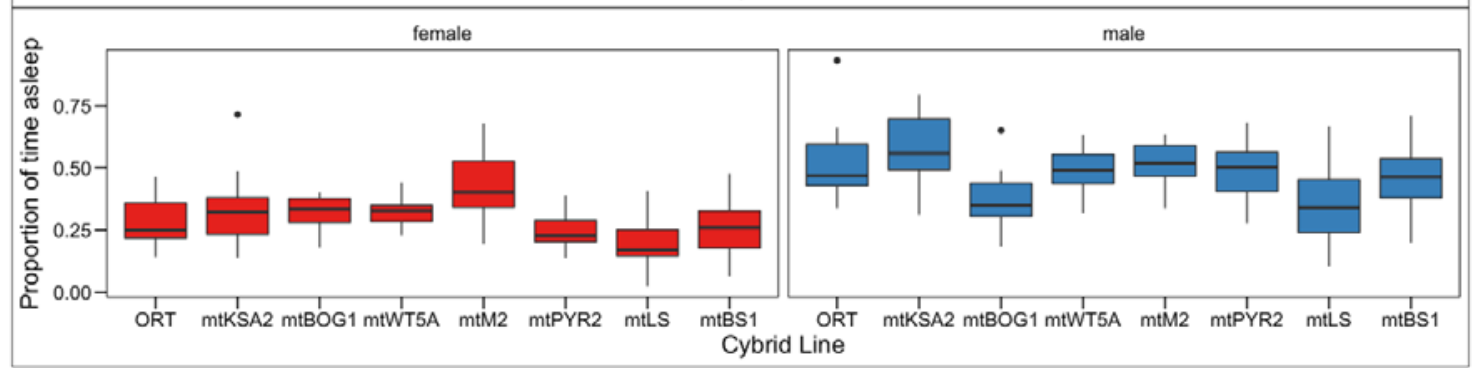

212 Figure 2. Mitochondrial haplotype effect. Locomotor activity and sleep in cybrid females and

213 males. Each mtDNA variant was introgressed onto the ORT nuclear background (ORT also

214 included here to allow direct comparisons). A) total number of activity events recorded over

215 three days. B) the total number of activity events recorded when flies were not asleep. C) The

216 proportion of time that flies were determined to be asleep, defined as 5 min of inactivity, See Fig

217 S2 for individual actograms and Table 1 for details of each mitochondrial haplotype. See Table 2

218 for outputs of statistical models. 


\begin{tabular}{lrrrrrr}
\hline & \multicolumn{2}{c}{ Total Activity Count } & \multicolumn{2}{c}{ Proportion asleep } & \multicolumn{2}{c}{ Mean awake activity } \\
\cline { 2 - 7 } Original Lines & $\chi^{2}$ & $\mathrm{p}$ & $\chi^{2}$ & $\mathrm{p}$ & $\chi^{2}$ & $\mathrm{p}$ \\
Line & & & & & & \\
Sex & 320.74 & $<0.001$ & 12808.10 & $<0.001$ & 10.56 & 0.159 \\
Line $\times$ Sex & 3.79 & 0.052 & 1063.30 & $<0.001$ & 9.40 & 0.002 \\
Cybrid lines & 59.90 & $<0.001$ & 1943.80 & $<0.001$ & 16.48 & 0.021 \\
Line & & & & & & \\
Sex & 40.80 & $<0.001$ & 2563.42 & $<0.001$ & 47.32 & $<0.001$ \\
Line $\times$ Sex & 134.56 & $<0.001$ & 5683.25 & $<0.001$ & 45.47 & $<0.001$ \\
\hline Coevolved vs. & 21.95 & 0.003 & 904.27 & $<0.001$ & 20.11 & 0.005 \\
Novel & & & & & & \\
Type & 18.62 & $<0.001$ & 10.42. & 0.0012 & 8.22 & $<0.001$ \\
Sex & 75.59 & $<0.001$ & 4985.67 & $<0.001$ & 0.001 & 0.975 \\
Type $\times$ Sex & 50.99 & $<0.001$ & 720.34 & $<0.001$ & 27.70 & $<0.001$ \\
\hline
\end{tabular}

Table 2. Summaries of test statistics for fixed effects in linear models. See methods for model

222 details.

223

224 Haplogroup specific mtDNA variation can be seen in the activities of cybrid females

225 Based on the mtDNA coding region variation the eight mtDNA variants form two distinct

226 haplogroups with a set of few common replacement variants present in haplogroup I in

227 OXPHOS complexes I (ND1; V190M, ND2; I277L, ND5; M502I) and V (ATP6; S538P and

228 M559V) (Salminen et al. 2017). Haplogroup I contains the haplotypes mtORT, mtKSA2,

229 mtBOG1, mtWT5A and mtM2, as the haplogroup II contains the haplotypes mtPYR2, mtLS

230 and mtBS1 (Table 1). Haplogroup division did not cause clear differences in the sleep-wake

231 activities when the haplogroup specific mtDNA variants were present in their coevolved nuclear

232 backgrounds and the sex differences that were observed earlier with the coevolved lines were

233 decreased (Table 3, Figure 3. upper row). However, when the haplogroup I and II mtDNA

234 variants were placed on a novel common nuclear background in the cybrid lines, we observed 
235 that haplogroup II females we more active than haplogroup I females (Table 3, Figure 3, lower

236 row A). Haplogroup II females were also more active when awake and slept for a smaller

237 proportion of time than haplogroup I females (Table 3, Figure 3, lower row B and C).
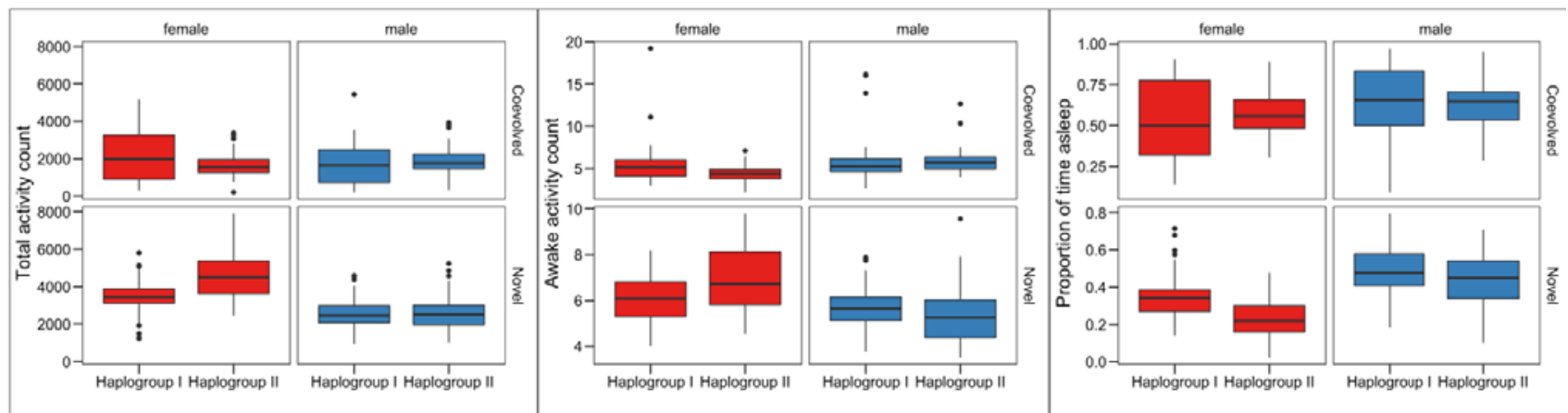

240 Figure 3. Haplogroup effect. Locomotor activities and sleep in coevolved and cybrid females

and males based on the mtDNA haplogroup division. A) Total activity counts over three days.

B) Activity of the flies when awake. C) The proportion of time the flies spent sleeping.

\section{Breaking up co-evolved mito-nuclear combinations affects activity and sleep patterns}

245 As naturally occurring mito-nuclear genome combinations have co-adapted locally over time, we

246 expected that disruption of these combinations could result in maladaptive effects. We also

247 expected that these effects might be more severe, or more variable in males, as postulated under

248 the mother's curse hypothesis (65-68). Both male and female cybrid flies harbouring novel mito-

249 nuclear combinations were significantly more active than co-evolved flies, although the extent of

250 this variation differed between sexes and was more prominent in females (Table 2 'Line x Sex'

251 effect; Figure 4A). This shift is partially due to the Oregon RT nuclear background, as shown it

252 Figure 1A ORT females had the highest total activity counts when compared to other co-

253 evolved lines. Part of the increase in total activity in cybrids was driven by increase in the awake

254 activity level, though this was the case only in females (Table 2 'Line x Sex' effect; Figure 4B).

255 However, the largest driver of the increased total activity in both male and female cybrid flies is 
256 that these spent a significantly lower proportion of their time asleep compared to the co-evolved

257 lines, the extent of this difference was slightly larger in females (Table 2 'Line x Sex' effect;

258 Figure 3C) and again, this is largely due to ORT nuclear background normalizing the variation of

259 the cybrid lines when compared the co-evolved lines (Fig. 1C, ORT females spent the least time

260 asleep).

261

262

263

Haplogroup effect

Coevolved Lines

Haplogroup

Sex

Haplogroup $\times$ Sex

Cybrid lines

Haplogroup

Sex
Haplogroup $\times$ Sex

Total Activity

$\chi^{2}$

0.78

3.07

7.77

0.05

$<0.001$

3.99

37.54

$<0.001$

15.53

0.0003

12.74

0.05

18.00

$<0.001$

0.24

1.66

0.197

Table 3. Summaries of test statistics for fixed effects in testing the effect of haplogroup and sex on activity and sleep in coevolved and cybrid lines. See Figure 3 and methods for model details.
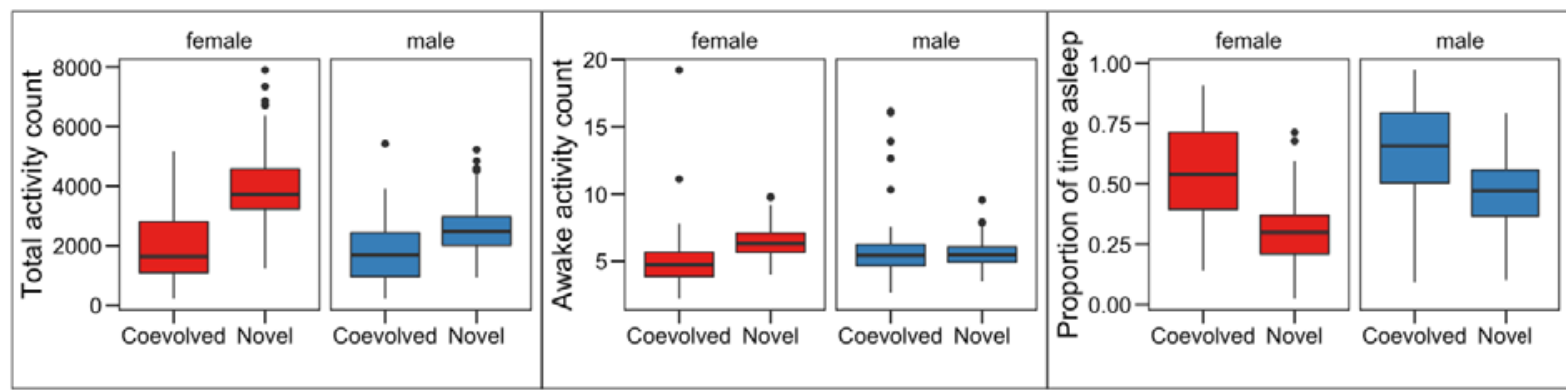

Figure 4. A comparison of Coevolved and Novel Mito-nuclear associations. A) total number of activity events recorded over 3 days. B) the total number of activity events recorded when flies were not asleep. C) The proportion of time that flies were determined to be asleep, 


\section{Cybrid activity levels are positively correlated with mtDNA copy number}

274 We utilized an earlier published mtDNA copy number data collected from the same co-evolved

275 and cybrid lines as studied in this manuscript (37). Salminen et al. (2017) measured mtDNA copy

276 number separately from females and males from various timepoints after eclosion. For our

277 purposes we selected 3-days post eclosion mtDNA copy number data as this was the closest

278 match with the age of most the flies used in our activity measurements. Our aim was to test the

279 correlation between mtDNA copy number and activity levels of the co-evolved and cybrid

280 females and males. When mtDNA copy number was correlated with the total activity counts of

281 the cybrid lines, we saw a significant positive correlation in cybrid males and similar but non-

282 significant trend in cybrid females, indicating that with increasing mtDNA copy number levels

283 the total activity of the flies also increased (Fig. 5A, right panel). We also saw a weak positive

284 trend between awake activity levels and mtDNA copy number (Fig. 5B, right panel) and a weak

285 negative trend between mtDNA copy number and sleep (Fig. 5C). In co-evolved lines we did

286 not detect evidence of a significant correlation between mtDNA copy number and activity or

287 sleep (Figure 5A-C, left panel). 

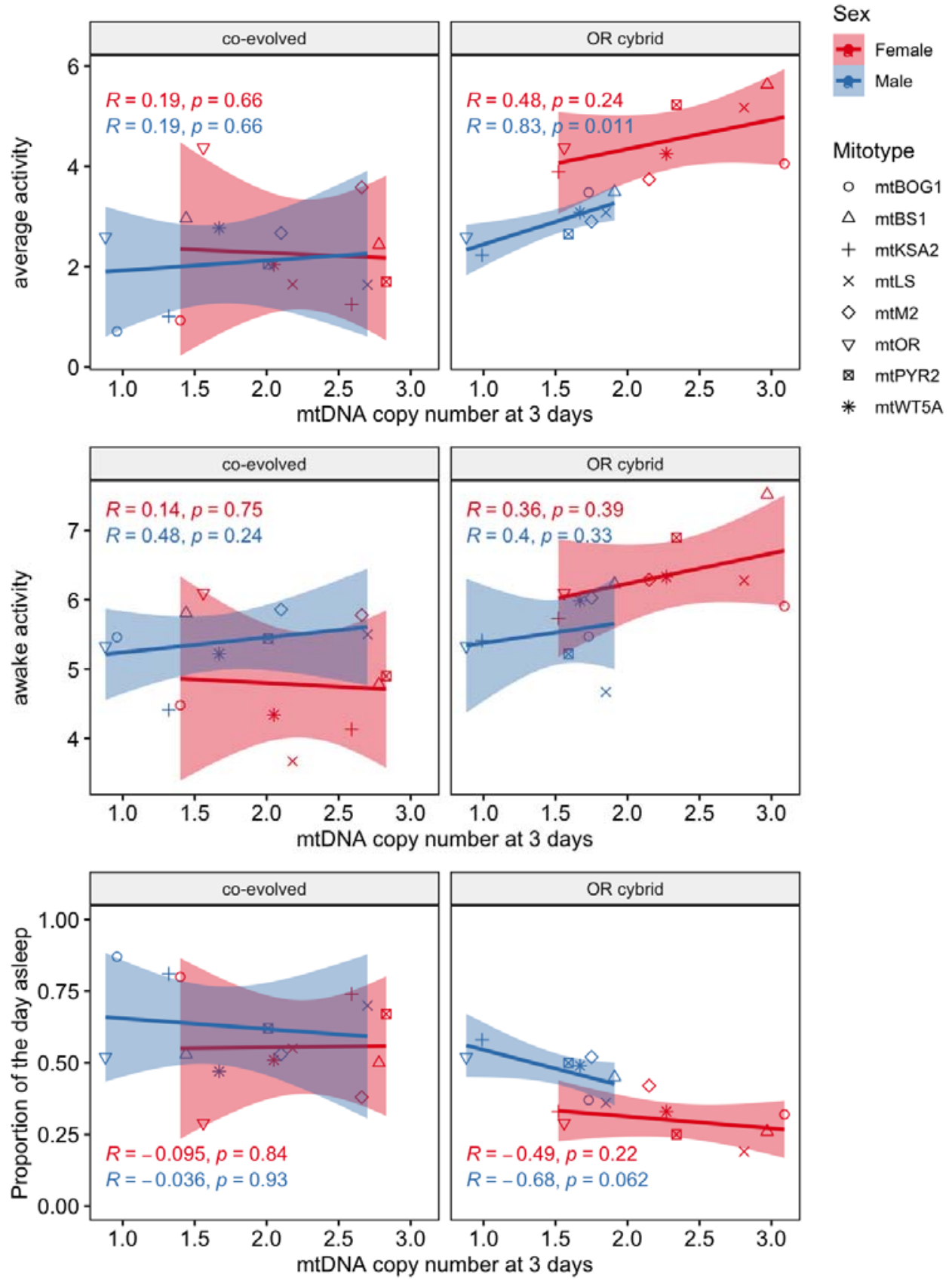

290 Figure 5. mtDNA copy number correlations between measured traits. Spearman's rank

291 correlations between average mtDNA copy number and averages of a) total number of activity

292 events, b) total number of activity events when the flies were awake and c) proportion of time

293 the flies were determined to be asleep measured from co-evolved (left panel) and cybrid (right

294 panel) females and males. 


\section{Discussion}

297 Mitochondrial dysfunction commonly manifests in phenotypes related to energy-demanding tissues, such as muscles, and movement disorders are a major feature of mitochondrial diseases

299 (53). Drosophila can be employed as a model for as movement disorders, and its locomotive

300 behaviours include larval crawling, adult walking, climbing and flight. These behaviours can be

301 used to evaluate muscle behaviour as a proxy of e.g. mitochondrial performance. Furthermore,

302 periods when the are immobile for longer than five-minutes, are typically considered sleeping

303 (39). Sleep deprivation has been shown to be connected to mitochondrial bioenergetics and to

304 cause mitochondrial dysfunction in Drosophila (54). However, investigation of sleep as a

305 consequence of mitochondrial dysfunction appears to be an understudied aspect of

306 mitochondrial disease (55). It is also unclear how retrograde signalling from mitochondria to

307 nucleus affects sleep-wake cycles, and especially the role of naturally occurring mtDNA variation

308 in these traits.

310 Studying the effect of mtDNA variation is challenging as it is necessary to disentangle the effects

311 of the mitochondrial genome from the effect of the nuclear genome. This is feasible with the

312 Drosophila cybrid model and here we have focused on studying the effect of mtDNA variation

313 and mito-nuclear interactions on locomotion activity and sleep. We addressed three questions by

314 separately quantifying the contributions of the mitochondrial and nuclear genomes to activity

315 and sleep phenotypes. First, we asked how variation in both the nDNA and mtDNA affects

316 sleep and activity. Second, by isolating mtDNA variants on a common nuclear background we

317 investigated how variation in mtDNA affects sleep and activity. Finally, we assessed how

318 breaking up co-evolved mito-nuclear genetic interactions affected the sleep and activity.

320 Locomotor activity and the proportion of time spent asleep were first measured from eight wild 321 type Drosophila strains with co-evolved mito-nuclear combination adapted to their local 
322 environment. These strains showed variation in their sleep-wake patterns and in general the

323 females were found to be more active overall; males exhibited a higher waking activity but slept

324 for a larger proportion of time. We next aimed to test if mtDNA contributed to this variation

325 between lines. The eight studied mtDNA genomes can be subdivided into two haplogroups

326 based on their genetic variation (37). These haplogroups differed in the amino acid replacements

327 present in OXPHOS complex I genes ND1, ND2 and ND5, as well as two variants from

328 complex V gene ATP6 (37). Based on these common replacement variants, haplogroup II

329 contained strains of European origin, whereas haplogroup I contained strains from different

330 continents (37). Interestingly, haplogroup II females were shown to have higher total activity

331 levels and spent less time in sleeping when compared to haplogroup I females. The same was not

332 observed with males. It is unclear how these patterns might have arisen in nature, however

333 further experimentation is required to examine of these patterns are a result of local adaptation

334 or just due to drift.

336 As the eight cybrids also possess mitochondrial haplotype-specific mutations, we examined the

337 effect of these to locomotion and sleep, when introgressed into common nuclear background in

338 the cybrid lines. In general, we were able to see differences in the amounts of activity and sleep,

339 in both females and males, brought upon by mtDNA variation. Cybrid females were more active

340 than males, sleep less and have higher waking activity. It is difficult to say if specific mtDNA

341 mutations are causing the seen variation, as most of these mtDNA genomes contain more than

342 one source of variation, i.e replacement variants in the protein coding genes, synonymous SNPs,

343 indels in the tRNA and rRNA genes and also length variation in the non-coding $\mathrm{A}+\mathrm{T}$ region

344 (37). Mitovariant mtM2, the only variant that is originally from Australia, contains the most

345 unique replacement variants when compared to the other mitovariants (Table 1). mtM2 was also

346 one of the lowest activity strains, and females especially spent more time sleeping when

347 compared to the other cybrids. This might be due to altered interactions between mitochondrial 
348 and nuclear gene products (56). M2 flies which have the original nuclear background actually

349 appear to exhibit relatively high activity and low amount of sleep. Disruption of naturally

350 occurring mito-nuclear combinations can result in interruption of precise interactions, leading to

351 decreased mito-nuclear cooperation and a reduction in fitness (36).

352

mtKSA2 is the only A frican variant, and it possesses two unique amino acid replacement variants

in OXPHOS cIII (CYTB) and cIV (COIII) when compared to other cybrids (37). mtKSA2 mutation in mtKSA2 may partially explain the lower activity rates when compared to other

362 strains.

mtKSA2 is also associated with low mtDNA copy number in ORT nuclear background when compared to other cybrid lines (37). Since mtDNA copy number has been shown to be associated with other measures of fitness in Drosophila including fertility and longevity (58) and with development time and weight (37) of the same co-evolved and cybrid lines as studied here, we hypothesised that it might also be associated with variation in activity levels. mtDNA copy number is sexually dimorphic in Drosophila, with females of most strains tending to exhibit a higher copy number than males $(37,58)$, and is also affected by the age of the flies, sex

371 specifically (37). Sleep and activity are also shown to be sexually dimorphic traits in Drosophila $372(39,59)$. In our study we saw that in both co-evolved and cybrid strains the females were more 373 active overall and slept less, as shown also with previous Drosophila sleep and activity research 
374 (60). These factors motivated us to analyse whether the earlier measured mtDNA copy number

375 levels of the co-evolved and cybrid females and males correlate with the traits measured in this

376 manuscript. We expected that higher activity and lower sleep exhibited by female flies may be at

377 least partly due to their higher mtDNA copy number. This was the case with the cybrid lines, but

378 was not seen with the co-evolved lines. The strongest positive correlation was found in cybrid

379 males between mtDNA copy number and mean activity levels. This data shows that in

380 controlled nuclear background the mtDNA effect on both mtDNA copy number and activity

381 levels have a stronger penetrance compared to co-evolved lines, where nuclear variation might

382 mask the effect of mtDNA variation on copy number and activity. In addition, the low mtDNA

383 copy number exhibited by nOR mtKSA2 among the cybrids is likely responsible for the strain's

384 reduced activity in some degree.

386 Comparison of the co-evolved mito-nuclear strains with the novel mito-nuclear strains allowed

387 investigation into the effects of mito-nuclear epistatic interactions and the effects of breaking up

388 possible co-evolved mito-nuclear gene complexes on organismal fitness. Previous studies have

389 demonstrated that disrupting these interactions can lead to decreased OXPHOS function (37,

390 61). Specifically, cytochrome c oxidase activity has been shown to be reduced when an mtDNA

391 haplotype is combined with a more distant nuclear background rather than its original nuclear

392 background in copepod Tigriopus californicus (62). Mito-nuclear incompatibilities can lead to

393 reduction in fitness (63-65), and maintaining normal levels of activity is beneficial to fitness to

394 allow, for example foraging and mating to take place (59). Short sleep duration in Drosophila has

395 previously been shown to be associated with poor memory (66), and reduced longevity (67, 68).

396 Therefore, it is likely that the altered sleep and activity patterns exhibited by cybrid strains are

397 likely to equate to a reduction in fitness, supporting the theory of mito-nuclear co-adaptation. 
399 Natural selection is blind to deleterious mitochondrial phenotypes which manifest in males as

$400 \mathrm{mtDNA}$ is maternally inherited (69-71). This can indicate that variation which persists in

$401 \mathrm{mtDNA}$ is more likely to cause greater phenotypic divergence within males and more likely to

402 cause deleterious effects in males than in females $(69,71)$. Although studies exist which provide

403 evidence in support of this theory, known as the mother's curse $(33,72)$, based on our results, it

404 does not appear that the effect on sleep or activity in males is more prominent than in females

405 when breaking up the co-evolved combinations, and does not provide evidence in support of the

406 mother's curse.

407

408 Drosophila sleep and activity research will enable further understanding of the causes of

409 abnormal sleep and activity patterns in humans and the role of mitochondrial variation in these

410 traits. Further studies of mito-nuclear interactions and $\mathrm{mtDNA}$ variation are essential in

411 recognizing and eventually preventing mito-nuclear mismatches that might occur during

412 mitochondrial replacement theory.

413

\section{Acknowledgements}

415 We thank Angela Reid and Lucinda Rowe and the Ashworth Media team for fly medium

416 preparation. Lucy Anderson was supported by the Genetics Honours Program, School of

417 Biological Sciences, University of Edinburgh. This work was partially supported by a

418 Chancellor's Fellowship (University of Edinburgh), a Branco Weiss Fellowship (Society in

419 Science - Zürich, https://brancoweissfellowship.org/) and a Leverhulme Trust Research Grant

420 (RPG-2018-369), all awarded to Pedro Vale. 


\section{References}

423 1. Chandel Navdeep S. Evolution of Mitochondria as Signaling Organelles. Cell 424 metabolism. 2015(0).

425 2. Vega-Naredo I, Loureiro R, Mesquita KA, Barbosa IA, Tavares LC, Branco AF, et al. 426 Mitochondrial metabolism directs stemness and differentiation in P19 embryonal carcinoma 427 stem cells. Cell Death Differ. 2014;21(10):1560-74.

428 3. Wang C, Youle RJ. The Role of Mitochondria in Apoptosis*. Annual Review of 429 Genetics. 2009;43(1):95-118.

430 4. Salminen TS, Vale PF. Drosophila as a Model System to Investigate the Effects of 431 Mitochondrial Variation on Innate Immunity. Front Immunol. 2020;11:521.

432 5. Gray MW, Burger G, Cedergren R, Golding GB, Lemieux C, Sankoff D, et al. A 433 Genomics Approach to Mitochondrial Evolution. Biol Bull. 1999;196(3):400-3.

434 6. Gray MW, Burger G, Lang BF. Mitochondrial evolution. Science. 1999;283(5407):147643581.

436 7. Lang BF, Gray MW, Burger G. Mitochondrial genome evolution and the origin of 437 eukaryotes. Annu Rev Genet. 1999;33:351-97.

438 8. Anderson S, Bankier AT, Barrell BG, de Bruijn MH, Coulson AR, Drouin J, et al. 439 Sequence and organization of the human mitochondrial genome. Nature. 1981;290(5806):457-65.

4409 9. Robin ED, Wong R. Mitochondrial DNA molecules and virtual number of mitochondria 441 per cell in mammalian cells. Journal of Cellular Physiology. 1988;136(3):507-13.

442 10. Hill GE, Havird JC, Sloan DB, Burton RS, Greening C, Dowling DK. Assessing the 443 fitness consequences of mitonuclear interactions in natural populations. Biol Rev Camb Philos 444 Soc. 2019;94(3):1089-104.

445 11. Smeitink J, van den Heuvel L, DiMauro S. The genetics and pathology of oxidative 446 phosphorylation. Nat Rev Genet. 2001;2(5):342-52.

447 12. Schon EA, DiMauro S, Hirano M. Human mitochondrial DNA: roles of inherited and 448 somatic mutations. Nat Rev Genet. 2012;13(12):878-90.

449 13. DiMauro S, Schon EA. Mitochondrial respiratory-chain diseases. N Engl J Med. $450 \quad 2003 ; 348(26): 2656-68$.

451 14. Wallace DC. Mitochondrial DNA sequence variation in human evolution and disease. 452 Proc Natl Acad Sci U S A. 1994;91(19):8739-46.

453 15. Salminen TS, Cannino G, Oliveira MT, Lillsunde P, Jacobs HT, Kaguni LS. Lethal 454 Interaction of Nuclear and Mitochondrial Genotypes in Drosophila melanogaster. G3 455 (Bethesda). 2019;9(7):2225-34.

456 16. Ganguly-Fitzgerald I, Donlea J, Shaw PJ. Waking experience affects sleep need in 457 Drosophila. Science. 2006;313(5794):1775-81. 
458 17. Trivedi MS, Holger D, Bui AT, Craddock TJA, Tartar JL. Short-term sleep deprivation 459 leads to decreased systemic redox metabolites and altered epigenetic status. PLoS One. $460 \quad 2017 ; 12(7): \mathrm{e} 0181978$.

461 18. Shaw PJ, Tononi G, Greenspan RJ, Robinson DF. Stress response genes protect against 462 lethal effects of sleep deprivation in Drosophila. Nature. 2002;417(6886):287-91.

463 19. Rechtschaffen A, Bergmann BM, Everson CA, Kushida CA, Gilliland MA. Sleep 464 deprivation in the rat: X. Integration and discussion of the findings. Sleep. 1989;12(1):68-87.

465 20. Potdar S, Sheeba V. Lessons from sleeping flies: insights from Drosophila melanogaster 466 on the neuronal circuitry and importance of sleep. J Neurogenet. 2013;27(1-2):23-42.

467 21. Harbison ST, McCoy LJ, Mackay TF. Genome-wide association study of sleep in 468 Drosophila melanogaster. BMC Genomics. 2013;14:281.

469 22. Videlier M, Rundle HD, Careau V. Sex-Specific Among-Individual Covariation in 470 Locomotor Activity and Resting Metabolic Rate in Drosophila melanogaster. Am Nat. $471 \quad 2019 ; 194(6): \mathrm{E} 164-\mathrm{E} 76$.

472 23. Filler K, Lyon D, Bennett J, McCain N, Elswick R, Lukkahatai N, et al. Association of 473 Mitochondrial Dysfunction and Fatigue: A Review of the Literature. BBA Clin. 2014;1:12-23.

474 24. Bar S, Prasad M, Datta R. Neuromuscular degeneration and locomotor deficit in a $475<$ em $>$ Drosophila $</$ em $>$ model of mucopolysaccharidosis VII is attenuated by treatment with 476 resveratrol. Disease Models \&amp; Mechanisms. 2018;11(11):dmm036954.

477 25. Ghimire S, Kim MS. Enhanced Locomotor Activity Is Required to Exert Dietary 478 Restriction-Dependent Increase of Stress Resistance in Drosophila. Oxid Med Cell Longev. 479 2015;2015:813801-.

480 26. Ramezani RJ, Stacpoole PW. Sleep disorders associated with primary mitochondrial 481 diseases. J Clin Sleep Med. 2014;10(11):1233-9.

482 27. Fogle KJ, Mobini CL, Paseos AS, Palladino MJ. Sleep and circadian defects in a 483 Drosophila model of mitochondrial encephalomyopathy. Neurobiol Sleep Circadian Rhythms. 484 2019;6:44-52.

485 28. Holmbeck MA, Donner JR, Villa-Cuesta E, Rand DM. A Drosophila model for mito486 nuclear diseases generated by an incompatible interaction between tRNA and tRNA synthetase. 487 Dis Model Mech. 2015;8(8):843-54.

488 29. Hoekstra LA, Siddiq MA, Montooth KL. Pleiotropic Effects of a Mitochondrial-Nuclear 489 Incompatibility Depend upon the Accelerating Effect of Temperature in $490<$ em>Drosophila</em>. Genetics. 2013;195(3):1129-39.

491 30. Zhu C-T, Ingelmo P, Rand DM. G $\times \mathrm{G} \times \mathrm{E}$ for Lifespan in Drosophila: Mitochondrial, 492 Nuclear, and Dietary Interactions that Modify Longevity. PLOS Genetics. 2014;10(5):e1004354.

493 31. Camus MF, Dowling DK. Mitochondrial genetic effects on reproductive success: 494 signatures of positive intrasexual, but negative intersexual pleiotropy. Proc Biol Sci. 495 2018;285(1879). 
496 32. Rand DM, Haney RA, Fry AJ. Cytonuclear coevolution: the genomics of cooperation.

497 Trends Ecol Evol. 2004;19(12):645-53.

498 33. Camus MF, Clancy DJ, Dowling DK. Mitochondria, maternal inheritance, and male 499 aging. Curr Biol. 2012;22(18):1717-21.

500 34. Rand DM, Fry A, Sheldahl L. Nuclear-mitochondrial epistasis and drosophila aging: 501 introgression of Drosophila simulans mtDNA modifies longevity in D. melanogaster nuclear 502 backgrounds. Genetics. 2006;172(1):329-41.

503 35. Camus MF, O'Leary M, Reuter M, Lane N. Impact of mitonuclear interactions on life504 history responses to diet. Philos Trans R Soc Lond B Biol Sci. 2020;375(1790):20190416.

505 36. Mossman JA, Ge JY, Navarro F, Rand DM. Mitochondrial DNA Fitness Depends on 506 Nuclear Genetic Background in Drosophila. G3 (Bethesda). 2019;9(4):1175-88.

507 37. Salminen TS, Oliveira MT, Cannino G, Lillsunde P, Jacobs HT, Kaguni LS. 508 Mitochondrial genotype modulates mtDNA copy number and organismal phenotype in 509 Drosophila. Mitochondrion. 2017;34:75-83.

510 38. Nagarajan-Radha V, Aitkenhead I, Clancy DJ, Chown SL, Dowling DK. Sex-specific 511 effects of mitochondrial haplotype on metabolic rate in Drosophila melanogaster support 512 predictions of the Mother's Curse hypothesis. Philos Trans R Soc Lond B Biol Sci. $513 \quad 2020 ; 375(1790): 20190178$.

514 39. Shaw PJ, Cirelli C, Greenspan RJ, Tononi G. Correlates of sleep and waking in 515 Drosophila melanogaster. Science. 2000;287(5459):1834-7.

516 40. Nitz DA, van Swinderen B, Tononi G, Greenspan RJ. Electrophysiological correlates of 517 rest and activity in Drosophila melanogaster. Curr Biol. 2002;12(22):1934-40.

518 41. Hendricks JC, Finn SM, Panckeri KA, Chavkin J, Williams JA, Sehgal A, et al. Rest in 519 Drosophila Is a Sleep-like State. Neuron. 2000;25(1):129-38.

520 42. Crocker A, Sehgal A. Genetic analysis of sleep. Genes Dev. 2010;24(12):1220-35.

521 43. Lewis E. A new standard food medium. Drosophila information service. 1960;34(117):152255.

523 44. Chiu JC, Low KH, Pike DH, Yildirim E, Edery I. Assaying locomotor activity to study 524 circadian rhythms and sleep parameters in Drosophila. J Vis Exp. 2010(43).

525 45. Andretic R, Shaw PJ. Essentials of sleep recordings in Drosophila: moving beyond sleep 526 time. Methods Enzymol. 2005;393:759-72.

527 46. Vale PF, Jardine MD. Sex-specific behavioural symptoms of viral gut infection and 528 Wolbachia in Drosophila melanogaster. J Insect Physiol. 2015;82:28-32.

529 47. Team RC. R: A language and environment for statistical computing. R Foundation for 530 Statistical Computing. 2019.

531 48. Wickham H. ggplot2: Elegant Graphics for Data Analysis. Use R. 2009:1-212. 
532 49. Wickham H, Francois R, Henry L, Müller K. dplyr: A Grammar of Data Manipulation. R 533 package version 0801. 2019:1-212.

534 50. Bates D, Maechler, M., Bolkler, B. Imet: Linear mixed-effects models using S4 classes. R 535 package version 0999999-0 http://CRANR-projectorg/package=lme4. 2012.

536 51. Fox J, Weisberg S. An R Companion to Applied Regression, Second Edition. 2011.

537 52. Lemon J. Plotrix: a package in the red light district of R. R-News. 2016;6(4):8-12.

538 53. Ghaoui R, Sue CM. Movement disorders in mitochondrial disease. J Neurol. 539 2018;265(5):1230-40.

540 54. Rodrigues NR, Macedo GE, Martins IK, Gomes KK, de Carvalho NR, Posser T, et al. 541 Short-term sleep deprivation with exposure to nocturnal light alters mitochondrial bioenergetics 542 in Drosophila. Free Radic Biol Med. 2018;120:395-406.

543 55. Brunetti V, Della Marca G, Servidei S, Primiano G. Sleep Disorders in Mitochondrial 544 Diseases. Curr Neurol Neurosci Rep. 2021;21(7):30.

545 56. Pichaud N, Berube R, Cote G, Belzile C, Dufresne F, Morrow G, et al. Age Dependent 546 Dysfunction of Mitochondrial and ROS Metabolism Induced by Mitonuclear Mismatch. Front 547 Genet. 2019;10:130.

548 57. Andreu AL, Hanna MG, Reichmann H, Bruno C, Penn AS, Tanji K, et al. Exercise 549 Intolerance Due to Mutations in the Cytochrome b Gene of Mitochondrial DNA. New England 550 Journal of Medicine. 1999;341(14):1037-44.

551 58. Camus MF, Wolf JB, Morrow EH, Dowling DK. Single Nucleotides in the mtDNA 552 Sequence Modify Mitochondrial Molecular Function and Are Associated with Sex-Specific 553 Effects on Fertility and Aging. Curr Biol. 2015;25(20):2717-22.

554 59. Long TA, Rice WR. Adult locomotory activity mediates intralocus sexual conflict in a 555 laboratory-adapted population of Drosophila melanogaster. Proc Biol Sci. 2007;274(1629):310555612.

557 60. Isaac RE, Li C, Leedale AE, Shirras AD. Drosophila male sex peptide inhibits siesta 558 sleep and promotes locomotor activity in the post-mated female. Proc Biol Sci. 559 2010;277(1678):65-70.

560 61. Sackton TB, Haney RA, Rand DM. Cytonuclear coadaptation in Drosophila: disruption 561 of cytochrome c oxidase activity in backcross genotypes. Evolution. 2003;57(10):2315-25.

562 62. Willett CS, Burton RS. Environmental influences on epistatic interactions: viabilities of 563 cytochrome c genotypes in interpopulation crosses. Evolution. 2003;57(10):2286-92.

564 63. Clancy DJ. Variation in mitochondrial genotype has substantial lifespan effects which 565 may be modulated by nuclear background. Aging Cell. 2008;7(6):795-804.

566 64. Clancy DJ, Hime GR, Shirras AD. Cytoplasmic male sterility in Drosophila melanogaster 567 associated with a mitochondrial CYTB variant. Heredity (Edinb). 2011;107(4):374-6. 
568 65. Yee WKW, Sutton KL, Dowling DK. In vivo male fertility is affected by naturally 569 occurring mitochondrial haplotypes. Current Biology. 2013;23(2):R55-R6.

570 66. Bushey D, Huber R, Tononi G, Cirelli C. Drosophila Hyperkinetic mutants have reduced 571 sleep and impaired memory. J Neurosci. 2007;27(20):5384-93.

572 67. Bushey D, Hughes KA, Tononi G, Cirelli C. Sleep, aging, and lifespan in Drosophila. 573 BMC Neurosci. 2010;11:56.

574 68. Cirelli C, Bushey D, Hill S, Huber R, Kreber R, Ganetzky B, et al. Reduced sleep in 575 Drosophila Shaker mutants. Nature. 2005;434(7037):1087-92.

576 69. Frank SA. Evolution: mitochondrial burden on male health. Curr Biol. $577 \quad 2012 ; 22(18): R 797-9$.

578 70. Carnegie L, Reuter M, Fowler K, Lane N, Camus MF. Mother's curse is pervasive across 579 a large mitonuclear Drosophila panel. Evol Lett. 2021;5(3):230-9.

580 71. Gemmell NJ, Metcalf VJ, Allendorf FW. Mother's curse: the effect of mtDNA on 581 individual fitness and population viability. Trends Ecol Evol. 2004;19(5):238-44.

582 72. Ruiz-Pesini E, Lapena AC, Diez-Sanchez C, Perez-Martos A, Montoya J, Alvarez E, et al. 583 Human mtDNA haplogroups associated with high or reduced spermatozoa motility. Am J Hum 584 Genet. 2000;67(3):682-96. 
bioRxiv preprint doi: https://doi.org/10.1101/2021.10.30.464953; this version posted November 1, 2021. The copyright holder for this preprint

(which was not certified by peer review) is the author/funder, who has granted bioRxiv a license to display the preprint in perpetuity. It is made available under aCC-BY 4.0 International license.

586 Supplementary Figures and tables

587

588

589 Fig S1 - Actograms for original lines with coevolved nuclear and mitochondrial genomes

590

591 Fig S2 - Actograms for cybird lines with novel nuclear and mitochondrial combinations

592 


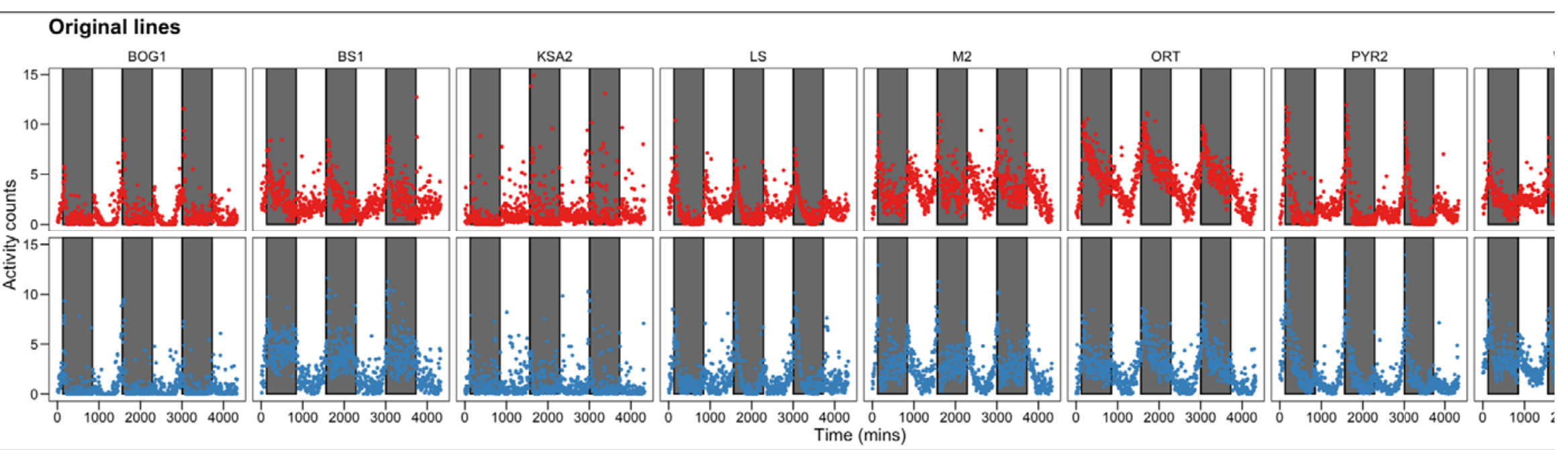

Fig S1 - Drosophila Activity Monitor (DAM) Activity counts (unique breaks of the infra-red beam) measured for each original fly line over a period

of three days. Females are shown in the top panel in red; males in the bottom panel in blue. Within each plot, the white vertical bars indicate periods 


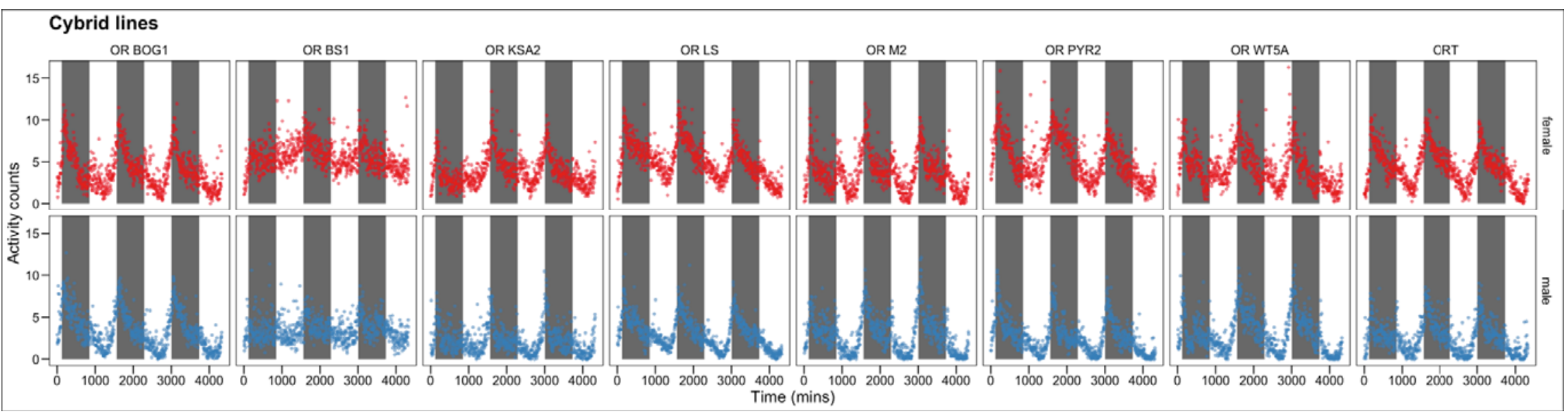

600

601

602

603

604

Fig S2 - Drosophila Activity Monitor (DAM) Activity counts over a period of three days (unique breaks of the infra-red beam) measured for each

cybrid fly line, where each mitochondrial haplotype has been introgressed onto the OR nuclear background. Females are shown in the top panel in red; males in the bottom panel in blue. Within each plot, the white vertical bars indicate periods of light, while the dark vertical bars indicate periods of dark when lights are turned off within the incubator. Details about each line can be found in Table 1 . Summary statistics for the activity patters can be found in

Figure 
bioRxiv preprint doi: https://doi.org/10.1101/2021.10.30.464953; this version posted November 1,2021 . The copyright holder for this preprint (which was not certified by peer review) is the author/funder, who has granted bioRxiv a license to display the preprint in perpetuity. It is made available under aCC-BY 4.0 International license.

\section{5}

